\title{
Titration of Power Output During Radiofrequency Catheter Ablation of Atrioventricular Nodal Reentrant Tachycardia
}

\author{
JONATHAN J. LANGBERG, MARK HARVEY, HUGH CALKINS, \\ RAFEL EL-ATASSI, STEVEN J. KALBFLEISCH, and FRED MORADY \\ From the Department of Internal Medicine, Division of Cardiology, The University of Michigan \\ Medical Center, Ann Arbor, Michigan
}

LANGBERG, J.J., ET AL.: Titration of Power Output During Radiofrequency Catheter Ablation of Atrioventricular Nodal Reentrant Tachycardia. Radiofrequency lesions in the anterior, superior aspect of the tricuspid annulus result in selective elimination of fast pathway function in patients with typical atrioventricular $(A V)$ nodal reentry tachycardia. This technique is simple and effective, but has been associated with a significant risk of inadvertent complete AV block. The purpose of this study was to compare the safety and effectiveness of two different techniques for radiofrequency catheter ablation of the fast $A V$ nodal pathway. Initially, a fixed power output was used at each target site. This method was compared retrospectively to a newer technique where power output was gradually incremented at each site. Radiofrequency power was initially applied at 10 watts for 10-15 seconds. If no junctional ectopy or a change in PR interval was seen, power output was incremented by 2 to 4 watts every 10 to 15 seconds up to a maximum of 30 watts. Thirty seven of $38(96 \%)$ patients treated using this incremental power output were cured of their AV nodal reentry tachycardia. None of these patients developed inadvertent complete AV block. In contrast, $92 \%$ of historic controls treated with a fixed power output between 20 and 30 watts achieved a primary success and nine of these $89(10 \%)$ historic controls developed inadvertent complete AV block $(P=0.04)$. There was no difference in the amplitudes of atrial, His, or ventricular electrograms at the effective sites between the two groups. Conclusions: (1) the anterior approach to radiofrequency catheter ablation of typical AV nodal reentry is associated with a significant risk of inadvertent complete AV block if a fixed power output is used; (2) starting at low power and gradually incrementing the output during radiofrequency energy application reduces the risk of complete AV block; (3) this incremental technique does not compromise efficacy. (PACE, Vol. 16, March, Part I 1993)

titration, radiofrequency catheter ablation, reentrant tachycardia

\section{Introduction}

Previous studies have shown that radiofrequency catheter ablation in the perinodal region can eliminate typical atrioventricular (AV) nodal reentry tachycardia without interrupting antegrade conduction. ${ }^{1-6}$ Lesions made in the anterior,

Address for reprints: Jonathan J. Langberg, M.D., University of Michigan Medical Center, 1500 East Medical Center Drive, B1 F245, Ann Arbor, MI 48109. Fax: (313) 936-7641.

Received August 17, 1992; revision October 8, 1992; accepted October 14, 1992. superior region of the tricuspid annulus, near the apex of the triangle of Koch, selectively eliminate fast AV nodal pathway function. ${ }^{1,7}$ While this technique has been shown to be effective, initial reports have suggested that it is associated with a significant risk of inadvertent complete AV block. $^{1-4}$

Studies in vitro and in animals have shown that lesions are created quickly after onset of radiofrequency energy application, with $50 \%$ of steadystate lesion size forming within 10-15 seconds. ${ }^{8,9}$ In addition, at any given site, there is a direct correlation between applied power and resultant lesion volume. ${ }^{8}$ These observations suggest that be- 
ginning at low output and increasing applied power in small increments during radiofrequency catheter ablation may result in slower lesion growth and increase the likelihood that the smallest effective lesion is created.

The purpose of this study was to compare the safety and effectiveness of two different techniques for radiofrequency catheter ablation of the fast AV nodal pathway in patients with typical AV nodal reentry tachycardia. Initially, a fixed power output was used at each target site. This method was compared retrospectively to a newer technique in which power output was gradually titrated to identify the minimum effective power output.

\section{Methods}

\section{Patient Population}

Between August 1989 and January 1992, 127 patients underwent radiofrequency catheter ablation for typical AV nodal reentry tachycardia using the anterior approach. Subsets of these patients have been described in earlier reports. ${ }^{1,7} \mathrm{~Pa}-$ tients who underwent ablation using the posterior approach or who had concomitant ablation of an extranodal accessory pathway were excluded from the analysis. The mean age of the patients was 51 \pm 18 years and 78 of the patients were women. Paroxysmal supraventricular tachycardia had been present for a mean of $17 \pm 15$ years and a mean of $2.9 \pm 1.8$; antiarrhythmic drug trials had been ineffective before ablation was attempted.

\section{Electrophysiological Test}

This study was performed under a protocol approved by the Human Research Committee at the University of Michigan and all patients gave written consent. All patients had a baseline electrophysiology test in order to define antegrade and retrograde AV nodal conduction and refractoriness and to confirm the presence of typical AV nodal reentry tachycardia using previously described techniques. ${ }^{7}$ The following criteria were sought to support the diagnosis of AV node reentry tachycardia: (1) tachycardia induction associated with a critical degree of prolongation of the $\mathrm{AH}$ interval; (2) atrial activation during tachycardia that was coincident with or that closely $1<50$ msec) followed ventricular activation; (3) failure of premature ventricular stimuli introduced during His bundle refractoriness to advance subsequent atrial activation. Other findings that were suggestive of AV node reentry tachycardia were the presence of discontinuous AV nodal function curves and tachycardia termination with a premature ventricular beat that did not conduct retrogradely to the atrium.

\section{Apparatus and Catheter Ablation Protocol}

Electrosurgical units that supplied continuous, unmodulated sine wave outputs were used for catheter ablation. Patients 1 through 76 were treated using a device with an operating frequency of $350 \mathrm{kHz}$ (Radionics RFG3B, Burlington, MA, USA) and patients 77 through 127 with a device that operated at $500 \mathrm{kHz}$ (EP Technologies, Inc., Mountain View, CA, USA). Applied power and impedance were continuously monitored during each energy application. Electrode catheters with a deflectable shaft and a distal electrode $4 \mathrm{~mm}$ in length were used for ablation (Mansfield Scientific, Watertown, MA, USA, and EP Technologies, Inc.). Radiofrequency energy was applied between the distal electrode of the ablation catheter and a large adhesive skin electrode placed over the left scapula.

Bipolar recordings from the ablation catheter were obtained with a bandpass of 50 to $500 \mathrm{~Hz}$ and a gain of $80 \mathrm{~mm}$ per $\mathrm{mV}$. The catheter was positioned at the site of the maximal His electrogram and then withdrawn several millimeters proximally and slightly anteriorly (Fig. 1). Electrograms at target sites typically had an atrial:ventricular electrogram ratio $>1$ and a His electrogram amplitude $<0.1 \mathrm{mV}$.

In patients 1 through 89, a fixed radiofrequency power output of $24.3 \pm 1.2$ watts (range 20 to 30 watts) was applied at each target site. If junctional ectopy or a change in PR interval was seen, then energy delivery was continued until the PR interval increased by $50 \%$ over baseline. If no effects were seen after 20 seconds, the ablation catheter was repositioned and the process repeated. Energy application was discontinued immediately if $2^{\circ}$ or $3^{\circ}$ block or a rise in impedance occurred. 

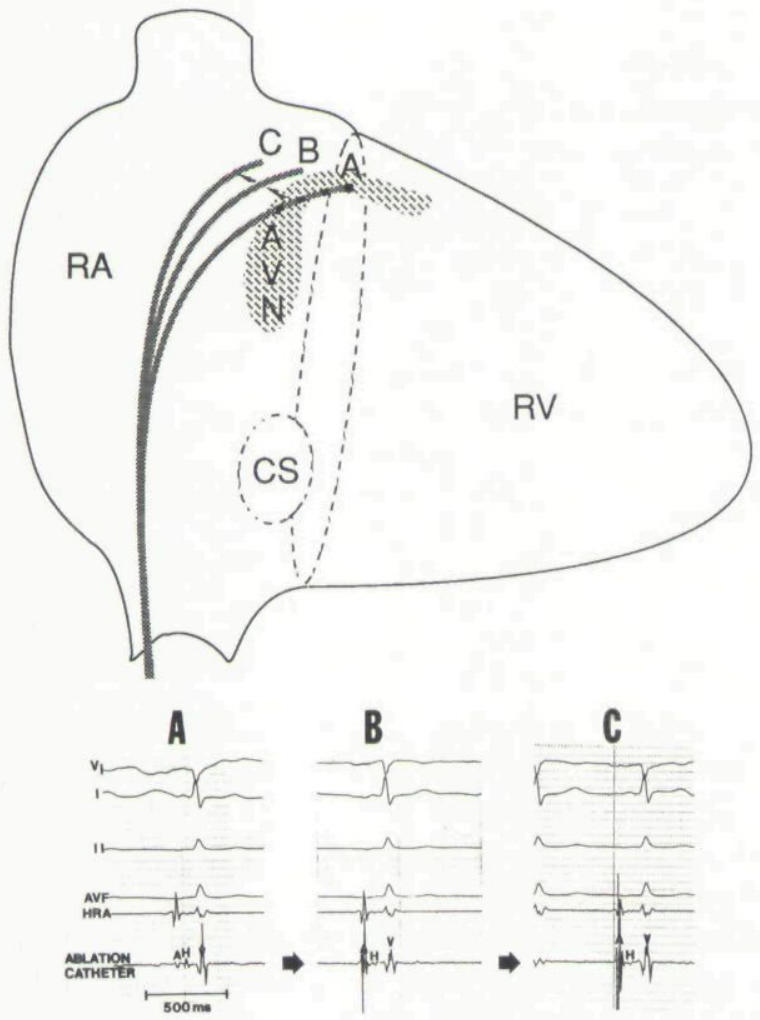

Figure 1. Manipulation of the ablation catheter and corresponding endocardial electrograms. In this diagram of the right anterior oblique projection, the ablation catheter is first positioned at the site of the maximal His bundle electrogram (A). At this site, the atrial electrogram $(A)$ is relatively small. As the catheter is withdrawn proximally and anteriorly (B), the His $(\mathrm{H})$ and ventricular $(\mathrm{V})$ electrograms diminish in size and the atrial electrogram grows. At the target site (C), the atrial electrogram is more than twice the amplitude of the ventricular electrogram and the His bundle deflection is barely visible. AVN = atrioventricular node; $\mathrm{CS}=$ ostium of the coronary sinus; HRA = recording from the high right atrium; $R A=$ right atrium; $R V=$ right ventricle. (Reprinted with permission. ${ }^{1}$ )

A different protocol was used in patients 90 through 127. At each target site, radiofrequency power was initially applied at 10 watts for 10 to 15 seconds. If no junctional ectopy or a change in PR interval was seen, power output was increased by 2 to 4 watts every 10 to 15 seconds up to a maximum of 25 to 30 watts. When junctional ectopy or a prolongation of the PR interval was observed, no further increments in power were made. Instead, power output was kept constant for an additional 15 to 20 seconds.

The endpoints for the ablation session were: (1) attenuation of retrograde fast pathway conduction as manifest by an increase in the retrograde AV nodal block cycle length and/or prolongation of the ventriculoatrial conduction time; (2) elimination of inducible AV nodal reentry. After a successful ablation, all patients had a repeat electrophysiology test before and during isoproterenol infusion sufficient to raise the resting heart rate by $>20 \%$ over baseline. Patients were also requested to return 3 months after the initial session for follow-up electrophysiology testing.

\section{Data Analysis}

Results were expressed as the mean \pm 1 standard deviation. Clinical, electrophysiological and ablation parameters in the cohort treated with a fixed power output were compared to those treated with incremental power output using Student's t-test for unpaired variables and Chi square analysis. A P value $<0.05$ was considered significant.

\section{Results}

\section{Baseline Clinical Characteristics}

The clinical characteristics of the cohort treated with incremental power output were not significantly different from those treated with a fixed power output (Table I).

As seen in Table II, baseline AV nodal function in the patients treated with the incremental approach was not significantly different from that in the group treated with a fixed power output, nor was there a difference in the rate of typical AV nodal reentry tachycardia induced at the baseline study.

The amplitudes of the atrial, His, and ventricular components of the bipolar electrograph at the effective site were measured in each patient. Amplitudes were comparable in the group treated with incremental power outputs and fixed power outputs (Table II). In particular, the mean His bundle electrogram amplitude was $68 \pm 68 \mu \mathrm{V}$ in the incremental group compared with $95 \pm 90 \mu \mathrm{V}$ in the control patients treated with a fixed power output $(\mathrm{P}=\mathrm{NS})$. 
Table I.

Clinical Features of Patients With AV Nodal Reentry Tachycardia Treated Using a Fixed Radiofrequency Power Output Versus An Incrementally Increasing Power Output

\begin{tabular}{lccc}
\hline & $\begin{array}{c}\text { Fixed } \\
\text { Power Output }\end{array}$ & $\begin{array}{c}\text { Incremental } \\
\text { Power Output }\end{array}$ & P Value \\
\hline Number of Subjects & 89 & 38 & NS \\
Gender & $32 \mathrm{M} / 57 \mathrm{~F}$ & $17 \mathrm{M} / 21 \mathrm{~F}$ & $\mathrm{NS}$ \\
Age & $51 \pm 19$ years & $53 \pm 18$ years & NS \\
Structural Heart Disease & $18 \%$ & $29 \%$ & $\mathrm{NS}$ \\
Duration of Symptoms & $17 \pm 14$ years & $17 \pm 16$ years & NS \\
Number of Previously Ineffective Drugs & $3.0 \pm 1.7$ & $2.6 \pm 1.9$ & \\
\hline
\end{tabular}

NS $=$ not significant.

The outcomes of radiofrequency catheter ablation in the two groups are compared in Table III. A mean of $6.8 \pm 4.9$ applications of energy were used in the patients treated with fixed power output compared with $6.3 \pm 4.4$ applications in those in whom titration was used. Procedure duration, defined as the time from insertion of hemostatic sheaths until completion of the postablation electrophysiology test was significantly shorter at 61 \pm 46 minutes in the subjects treated using the incremental approach compared with $88 \pm 37$ minutes in subjects in whom a fixed power output was used $(\mathrm{P}=0.008)$.

Primary success, defined as elimination of inducible AV node reentry with preservation of
1:1 conduction in sinus rhythm, was achieved in comparable proportions of both cohorts (Table III).

Inadvertent complete AV block was produced in 9 of the 89 patients in whom a fixed power output was used. The frequency of this complication did not appear to diminish with increasing experience; two cases occurred in patients 1 through 30 , three in patients 31 through 60 , and four in patients 61 through 89. In contrast, none of the subsequent 38 patients treated using the incremental technique developed complete AV block $(\mathrm{P}=$ 0.042).

During a mean follow-up of $9.2 \pm 5.4$ months there were three recurrences of typical AV nodal reentry tachycardia. Two of these patients had

Table II.

Comparison of Baseline Electrophysiological Features of the Two Patient Groups

\begin{tabular}{lcrc}
\hline & $\begin{array}{c}\text { Fixed } \\
\text { Power Output }\end{array}$ & $\begin{array}{c}\text { Incremental } \\
\text { Power Output }\end{array}$ & P Value \\
\hline AH & $94 \pm 65 \mathrm{msec}$ & $88 \pm 23 \mathrm{msec}$ & $\mathrm{NS}$ \\
AV BCL & $365 \pm 83 \mathrm{msec}$ & $353 \pm 67 \mathrm{msec}$ & $\mathrm{NS}$ \\
VA BCL & $350 \pm 93 \mathrm{msec}$ & $338 \pm 66 \mathrm{msec}$ & $\mathrm{NS}$ \\
AVNRT CL & $382 \pm 75 \mathrm{msec}$ & $341 \pm 72 \mathrm{msec}$ & $\mathrm{NS}$ \\
Target site electrogram amplitudes & & & \\
$\quad$ Atrial & $1.3 \pm 0.85 \mathrm{mV}$ & $0.85 \pm 0.50 \mathrm{mV}$ & $\mathrm{NS}$ \\
$\quad$ His & $0.095 \pm 0.090 \mathrm{mV}$ & $0.068 \pm 0.068 \mathrm{mV}$ & $\mathrm{NS}$ \\
$\quad$ Ventricular & $0.78 \pm 0.60$ & $0.53 \pm 0.53 \mathrm{mV}$ & $\mathrm{NS}$ \\
\hline
\end{tabular}

$\mathrm{AH}=$ atrio-His interval; $\mathrm{AV}=$ atrioventricular; $\mathrm{AVNRT}=$ atrioventricular nodal reentry tachycardia; $\mathrm{BCL}=\mathrm{Wenckebach}$ block cycle length; $\mathrm{CL}=$ cycle length; $\mathrm{VA}=$ ventriculoatrial; $\mathrm{NS}=$ not significant. 
Table III.

Outcome of Radiofrequency Catheter Ablation of AV Nodal Reentry Tachycardia Using a Fixed Power Output Compared with an Incrementally Increasing Power Output

\begin{tabular}{|c|c|c|c|}
\hline & $\begin{array}{c}\text { Fixed } \\
\text { Power Output }\end{array}$ & $\begin{array}{l}\text { Incremental } \\
\text { Power Output }\end{array}$ & P Value \\
\hline Number of Subjects & 89 & 38 & \\
\hline Number of Radiofrequency Applications & $6.8 \pm 4.9$ & $6.3 \pm 4.4$ & NS \\
\hline${ }^{\star}$ Procedure Duration & $88 \pm 37 \mathrm{~min}$ & $61 \pm 46 \mathrm{~min}$ & NS \\
\hline${ }^{\star}$ Primary Success & $74 / 80(92 \%)$ & $37 / 38(96 \%)$ & NS \\
\hline Complete AV Block & $9 / 89(10 \%)$ & $0 / 38$ & 0.042 \\
\hline \multicolumn{4}{|c|}{ Results of Follow-up Electrophysiology Testing } \\
\hline $\mathrm{AH}$ & $134 \pm 74 \mathrm{msec}$ & $127 \pm 55 \mathrm{msec}$ & NS \\
\hline AV BCL & $297 \pm 63 \mathrm{msec}$ & $327 \pm 98 \mathrm{msec}$ & NS \\
\hline VA BCL & $360 \pm 78 \mathrm{msec}$ & $362 \pm 126 \mathrm{msec}$ & NS \\
\hline VA Dissociation & $41 \%$ & $28 \%$ & NS \\
\hline Recurrence & $12 / 8015 \%$ & $5 / 3714 \%$ & NS \\
\hline
\end{tabular}

* See text for definitions. Abbreviations as in Table II.

been treated with the fixed power output and one with the incremental approach. New high grade AV block was not observed during follow-up.

The target site electrogram did not appear to be predictive of high grade AV block in the cohort treated with a fixed power output. There was no difference in the amplitudes of the atrial, His, or ventricular electrograms at the nine sites that caused block compared with the 74 sites that were successful without block $(1.3 \pm 0.55,0.075 \pm$ $0.10,0.90 \pm 0.65 \mathrm{mV}$ vs $1.27 \pm 0.88,0.095 \pm$ 0.090, $0.75 \pm 0.60 \mathrm{mV}, \mathrm{P}=\mathrm{NS}$ ).

\section{Follow-Up Evaluation}

Immediately after ablation, follow-up testing revealed comparable effects on fast pathway function in both groups (Table III). Both groups of patients had an increase in the mean $\mathrm{AH}$ interval of approximately $40 \mathrm{msec}$. There was no significant change in antegrade AV nodal block cycle length after ablation. In contrast, retrograde conduction was significantly impaired, with an increase in the retrograde block cycle length or complete ventriculoatrial dissociation in both groups.

Tachycardia recurred either spontaneously or at the time of follow-up electrophysiological study in $12 / 80$ patients $(15 \%)$ successfully treated using a fixed power output and in 5/37 patients (14\%) treated with the incremental approach $(\mathrm{P}=\mathrm{NS})$.

\section{Discussion}

\section{Main Findings}

Results of this study suggest the following: (1) the anterior approach to radiofrequency catheter ablation of typical AV nodal reentry is associated with a significant risk of inadvertent complete AV block if a fixed power output is used; (2) this risk is independent of the "learning curve" for the procedure and cannot be predicted by the target site electrogram; (3) starting at low power and gradually titrating the output during radiofrequency energy application reduces the risk of complete AV block; (4) this incremental technique does not prolong the procedure nor does it compromise efficacy.

This study is limited by the retrospective nature of the comparison. The shorter procedure duration and the trend towards lower His electrogram amplitudes at the effective site in the 38 patients treated with the incremental technique compared to historic controls treated with a fixed power output suggests that the "learning curve" may have influenced the results. However, the in- 
cidence of inadvertent complete AV block did not diminish with increasing experience in the control patients. Therefore, it seems possible that the new energy delivery strategy may have contributed to the observed improvement in procedural safety.

\section{Comparison to Previous Studies and Clinical Implications}

Radiofrequency catheter ablation of AV node reentry using the anterior approach has been described in previous reports. ${ }^{1,2,4}$ The incidence of complete AV block in these series ranged from $8 \%$ to $21 \%{ }^{1,4}$ As in the current study, electrographic and energy delivery parameters did not appear to be useful predictors of subsequent AV block. ${ }^{7}$

Radiofrequency lesions made posteriorly, near the ostium of the coronary sinus, affect slow AV nodal pathway function. ${ }^{3-6}$ This posterior ap-

\section{References}

1. Lee MA, Morady F, Kadish A, et al. Catheter modification of the atrioventricular junction with radiofrequency energy for control of atrioventricular nodal reentry tachycardia. Circulation 1991; 83: 827-835.

2. Goy JJ, Fromer M, Schlaepfer, et al. Clinical efficacy of radiofrequency current in the treatment of patients with atrioventricular node reentrant tachycardia. J Am Coll Cardiol 1990; 16:418-423.

3. Kay GN, Epstein AE, Dailey SM, et al. Selective radiofrequency ablation of the slow pathway for the treatment of atrioventricular nodal reentrant tachycardia: Evidence for involvement of perinodal myocardium within the reentrant circuit. Circulation 1992; 85:1675-1688.

4. Jazayeri MR, Hempe SL, Sra JS, et al. Selective transcatheter ablation of the fast and slow pathways using radiofrequency energy in patients with atrioventricular nodal reentrant tachycardia. Circulation 1992; 85:1318-1328.

5. Haissaguerre M, Gaita F, Fischer B, et al. Elimination of atrioventricular nodal reentrant tachycardia using discrete slow potentials to guide application proach appears to be effective and does not result in prolongation of the $\mathrm{AH}$ interval in sinus rhythm. More importantly, target sites are most often several centimeters from the site of the maximal His electrogram, suggesting that the risk of complete AV block may be lower with the posterior approach than with the anterior approach. To date, no cases of high grade AV block have been reported following posterior lesions.

These studies, albeit preliminary, suggest that the posterior approach is the technique of choice for radiofrequency catheter ablation of typical AV nodal reentry. However, $3 \%$ to $12 \%$ of patients in these series could not be successfully ablated with posterior lesions. In these patients it may be necessary to use the anterior approach. Results of this study suggest that anterior lesions can be made safely if radiofrequency power is gradually incremented at each site.

of radiofrequency energy. Circulation 1992; 6: 2162-2175.

6. Jackman WM, Beckman JK, McClelland JH, et al. Treatment of supraventricular tachycardia due to atrioventricular nodal reentry by radiofrequency catheter ablation of slow-pathway conduction. N Engl J Med 1992; 327:313-318.

7. Calkins H, Sousa J, El-Atassi R, et al. Diagnosis and cure of the Wolff-Parkinson-White syndrome or paroxysmal supraventricular tachycardias during a single electrophysiologic test. N Engl J Med 1991; 324:1612-1618.

8. Wittkampf FHM, Hauer RNW, Robles de Medina E. Control of radiofrequency lesion size by power regulation. Circulation 1989; 80:962-968.

9. Haines DE, Watson DD. Tissue heating during radiofrequency catheter ablation: A thermodynamic model and obsevations in isolated perfused and superfused canine right ventricular free wall. PACE 1989; 12:962-976.

10. Blanck Z, Makal J, Hanson L, et al. Prediction of atrioventricular block during radiofrequency ablation of the fast pathway in atrioventricular nodal reentry. (abstract) Circulation 1991; 84:II-235. 
This document is a scanned copy of a printed document. No warranty is given about the accuracy of the copy. Users should refer to the original published version of the material. 